\title{
Optimum Allocation of Reactive Power in Real-Time Operation under Deregulated Electricity Market
}

\author{
Mahdi Rajabzadeh ${ }^{\dagger}$ and Masoud A. Golkar*
}

\begin{abstract}
Deregulation in power industry has made the reactive power ancillary service management a critical task to power system operators from both technical and economic perspectives. Reactive power management in power systems is a complex combinatorial optimization problem involving nonlinear functions with multiple local minima and nonlinear constraints. This paper proposes a practical market-based reactive power ancillary service management scheme to tackle the challenge. In this paper a new model for voltage security and reactive power management is presented. The proposed model minimizes reactive support cost as an economic aspect and insures the voltage security as a technical constraint. For modeling validation study, two optimization algorithm, a genetic algorithm (GA) and particle swarm optimization (PSO) method are used to solve the problem of optimum allocation of reactive power in power systems under open market environment and the results are compared. As a case study, the IEEE-30 bus power system is used. Results show that the algorithm is well competent for optimal allocation of reactive power under practical constraints and price based conditions.
\end{abstract}

Keywords: Ancillary Services, Deregulation, Reactive power Market, Voltage Stability criteria

\section{Introduction}

The primary objective of ISO is to match the electricity supply with demand. At the same time, ISO must control generation to the extent necessary to maintain reliability, optimize transmission efficiency, and maintain stability of participants, such as GenCo, TransCo and Discos is to maximize theirs own profit [1].

Reactive power management and control is a significant factor to support the system security and reliability. In a deregulated electricity market, active power has generally become a commodity, and reactive power has been treated as an ancillary service. And many of the deregulated electricity markets have established financial compensation mechanisms for reactive power services [2].

Adequate provision for reactive power is essential in power systems in order to ensure secure and reliable operation of power systems. Reactive power is tightly related to bus voltages throughout a power network, and hence reactive power services have a significant effect on system security. Insufficient reactive power supply can result in voltage collapse, which has been one of the reasons for some recent major blackouts [3].

Reactive power is supplied by several different sources, including transmission equipment (such as capacitors, reactors, Static VAR Compensators (SVCs) and static compensators), generators, and synchronous condensers. Reactive power does not travel over long distances at high line loadings due to significant losses on the wires. Thus, reactive

$\dagger$ Corresponding Author: K.N.Toosi University of Technology, Tehran, Iran. (Mahdi_rajabzadeh_0098@yahoo.com)

* K.N.Toosi University of Technology, Tehran, Iran. (Golkar@eetd.kntu.ac.ir)

Received 12 March 2009; Accepted 9 July 2009 power usually must be procured from suppliers near where it is needed. This factor limits the geographic scope of the reactive power market and, thus, the number of suppliers that can provide reactive power and the amount of competition at any place and time, at least in the short term before other suppliers can enter the market [4].

This paper focuses on and challenges the issues related to reactive power ancillary service procurement and pricing in competitive power market environments. Most existent plans for reactive power purchasing have two common aims; one of them is to create propellant in reactive generators in order to participate in reactive power market and appropriate coordination between economical and technical problems $[5,6]$. Early attempt of this approach similar to real power spot price theory can be found in [7]. But this model can not create a transparent competition between reactive generators and there is no attention to voltage stability. Li and David proposed a modified AC OPF model for reactive power wheeling rates analysis [8]. Bhattacharya and Zhong constructed a reactive bid curve of generator in [9] and further develop a competitive market mechanism based on it in [10]. However, as pointed out in [11], the application of marginal reactive price is not very practical due to its volatile and erratic behaviors. With these considerations cost-based reactive power pricing and management methods were proposed in $[11,12]$. However, there are two drawbacks in their proposed approaches, namely technical signals but not economic signals are used to determine generators' reactive power obligation and only reactive power amount, without considering the effects of the loading location on the costs, is used to charge reactive loads. As a result economic effectiveness is deteriorated [13]. In [6, 14-16] the real power market consequences are supposed to be steady and then the optimal 
reactive power flow is done, also all the reactive capacities are obliged to participate in market. Generators need reactive power in order to generate real power but this model pay money to generators in all operation conditions. Such a structure has many differences to competitive one. A model for simulation of voltage stability can be found in [17] but it did not discuss the determination of operating point by the use of voltage stability margin, also there is no warranty for the preservation of voltage stability. A model was proposed in [18] which minimize the active and reactive power generation cost simultaneously, since the active power generation cost is much bigger than reactive power generation cost, therefore the act of reactive generators will be neglected. There is no attention to voltage stability in this paper too.

This paper proposed a new model for the optimal allocation of the reactive power reserves in real-time operation. In the proposed method, the management of the reactive power reserves is proposed as an optimization problem. The main objective of the optimization is to minimize reactive support cost as well as to decrease the active power losses by rescheduling the reactive power injection of the reactive power supports. In this approach, the voltage stability margin is incorporated in to the power flow equations to maintain the power system in a secure operating state where a sudden load variation or even a contingency occurs. Optimal reactive power dispatch is obtained with the objective of minimizing reactive support cost. Then, a pricing structure including compensation to reactive sources and charge of reactive consumers is established with both technical feasibility and economic equitability taken into account. In this paper ISO, as a facilitator of reactive service, assures the total compensations to reactive suppliers equal to the revenues from the reactive power consumer. Based on analysis of generators' production cost of reactive power, a segmented bidding model of generators' reactive power generation is exerted [19]. The proposed method specifies the active and reactive power of generators and the reactive power of static VAR compensators and the variation of transformers tap changer as well as their cost. In this paper both active and reactive power flow is accomplished while optimization. Each reactive power supports proposes its reactive power capacity and its cost by a clear function so there are a transparent competition between reactive generators. The optimization problem is solved with both genetic algorithm (GA) and particle swarm optimization (PSO) method. Finally, the simulation results on a IEEE-30 bus power system are presented.

\section{Favorite Reactive Power Market Model}

The main subjects related with the reactive power management are: maintaining an adequate security level, defining correct economic signals, providing a simple and transparent structure, ensuring market equity, and avoiding additional charges on the final energy price. Reactive power planning and management in deregulated electricity market is Significant in three aspects. In long-term reactive power planning, system security is important in long-term voltage stability and the main goal is to determine the amount and placement of reactive resources to achieve voltage stability. Another is short-term reactive power planning and management. In this case it is supposed that the reactive resources are denoted thus the optimal allocation of reactive reserves in short-term is significant. And the last is reactive power management in Real-Time Operation. In which the reactive power capacity is available and the optimal uses of reactive resources for voltage stabilization in real-time operation is important.

By the inspection of reactive power market models, it is observable that the reactive power market structure should be able to prepare the necessary conditions for the independent reactive power generators to participate in the market and also should have the ability to adjust energy treaties when there is reactive power resources shortage or even utility technical constraints. The designed model should prepare the competition possibility for energy treaties stabilization. Reactive power market structure should consider the utility voltage stability in a proper manner and achieving an optimal point as a reactive power market consequence can not assure utility stability operation in realtime operation.

\section{Problem Formulation}

In the deregulated electricity market, concentration is on optimal reactive power purchasing in order to minimize management costs. Reactive power management in realtime operation is considered in this part. Active power generation quantity and situation, transmission system configuration and also the location of reactive power resources have the significant role in voltage stability. A new model for the optimal allocation of reactive power is proposed which considers the voltage stability restriction in the realtime operation besides voltage magnitude constraints.

\subsection{Objective Function}

The following objective function is formulated where ${ }_{Q_{g i}}$, $Q_{s l j}$ and $Q_{g i}^{\xi}$ are purchased reactive power of generators at bus $i^{\text {th }}$, static VAR compensators at bus $j^{\text {th }}$ and purchased reactive power of generators at bus $i$ with the load increase respectively; $\Delta P_{g i}$ is the active power participation of generators at bus $\mathrm{i}^{\text {th }}$ which compensate utility losses and ${ }_{\Delta T}$ is the change of transformation ratio.

$$
\begin{aligned}
& \operatorname{Min} J\left(Q_{g}, Q_{s h}, Q_{g}^{\xi}, \Delta P_{g}, \Delta T\right)=\sum_{i=1}^{N_{G}} \lambda_{g i}\left(Q_{g i}\right)+\sum_{j=1}^{N_{s h}} C_{s h j}\left(Q_{s h j}\right)+ \\
& +\sum_{k=1}^{N_{T}} C_{T k}\left(\Delta T_{k}\right)+\sum_{i=1}^{N_{G}} \tau_{g g i} \lambda_{g i}\left(Q_{g i}^{\xi}\right)+M C P \quad\left(\sum_{i=1}^{N_{G}}\left(P_{g i}^{0}+\Delta P_{g i}\right)-\sum_{l=1}^{N_{D}} P_{d l l}^{0}\right)
\end{aligned}
$$

In Eqn. (1) $P_{g i}^{0}$ and $P_{d l}^{0}$ are the active power outputs of generator $\mathrm{i}$ and the power consumption of consumer 1 
respectively which are obtained from electricity market; $\tau_{g q i}$ is the adjustment coefficient of reactive power cost increase while increasing in load; $M C P$ is the market clearing price in which electricity is purchased in order to compensate the active power losses. $N_{G}, N_{s h}, N_{T}$ and $N_{D}$ are the number of generator units, static VAR compensators, transformers and loads respectively. $\lambda_{g i}\left(Q_{g i}\right)$ and $C_{s h j}\left(Q_{s h j}\right)$ are the costs of reactive power support from the point of view of each generator or static VAR compensator respectively which their computational methods are discussed in subsequent part. $C_{T k}\left(\Delta T_{k}\right)$ is the cost function of adjustable ratio transformer $\mathrm{k}$, the fourth term is the cost of reactive power support while increasing in load and the final term represents the active power loss, which is procured at market clearing price.

\section{A. Reactive Power Bidding Model of Generators}

ISO operates the market of reactive power ancillary service according to the quoted price for reactive power of each generator unit and coordinates the reactive compensation devices and transformers belonged to power Grid Company to procure the needed reactive power. Because ISO cannot know exactly the reactive power generation cost of generator units, based on general characters of reactive power generation cost, the reactive power segmented bidding model of generator units is used there, that is to say, generator units join the reactive power market by bidding.

The reactive power generation costs of generator units are composed of investment cost, opportunity cost and operation and maintenance cost. The investment cost is reactive power capacity cost of generator units, and the opportunity cost equals to the reduced active power income because of the increased reactive power. The operation and maintenance cost aims mainly that when generator operates in leading phase, the temperature of the stator head increases, and the range of steady stability decreases, so the operators should strengthen the management of equipments, and this part of expenses should be compensated.

When generators in operating, the constraints of the stator winding temperature, the excitation winding temperature, the power of prime move, the temperature of stator head in leading phase and the stability in parallel compose the operation limit diagram of generator units, as shown in Fig. 1. The operation point of generator is limited within the area of A-N-H-D.

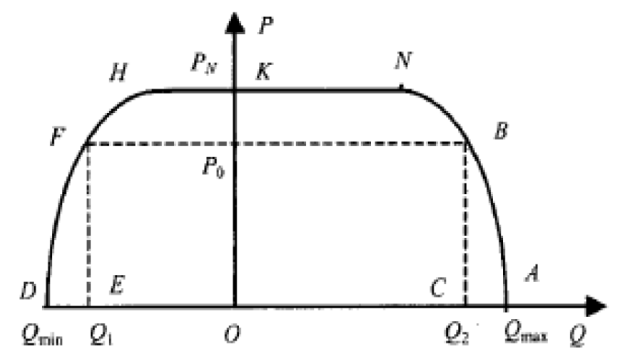

Fig. 1. Operation limit diagram of generator
Suppose that one generator unit gets $p_{0}$ active power by bidding, when the generator operates in area $0-\mathrm{K}-\mathrm{N}-\mathrm{B}-\mathrm{C}$, the active power will not decrease when the reactive power increases, so the reactive power cost mainly included investment cost. When the generator operates in area $\mathrm{C}-\mathrm{B}-\mathrm{A}$, it has to reduce its active power in order to increase reactive power, so the reactive power cost should include investment cost and reduced active power income. When operates in area $0-\mathrm{K}-\mathrm{H}-\mathrm{F}-\mathrm{E}$, the generator is in leading phase, so the reactive power cost should include investment cost and operation fare for leading phase. In the same way, when the generator operates in area D-F-E, the reactive power cost must include investment cost, opportunity cost and operation fare for leading phase.

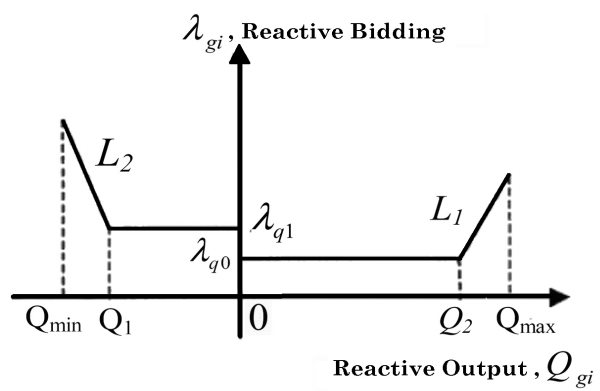

Fig. 2. Reactive power bidding curve of generator units

Based on above analysis, the reactive power-bidding curve of generator units is as in Fig. 2; and the corresponding reactive power-bidding model is shown in Eqn. (2). $Q_{\max }, Q_{\min }, Q_{1}, Q_{2}$ in Fig. 2 correspond to the corresponding value in Fig. 1. $\lambda_{q 0}$ Compensated the reactive power investment cost of generator, corresponding to the capacity price of reactive power, to encourage generator traders to invest reactive power and ensure system having enough reactive power resources. $\left(\lambda_{q 1}-\lambda_{q 0}\right)$ compensates the expenditure for generator in leading phase. Beeline $L_{1}$ compensates the investment cost and opportunity cost of reactive power, and $L_{2}$ compensates the investment cost and opportunity cost of reactive power and operation in leading phase.

$$
\lambda_{g i}\left(Q_{g i}\right)= \begin{cases}\lambda_{q 1}+\left(a^{\prime} Q_{g i}+b^{\prime}\right) & Q_{\min } \leq Q_{g i} \leq Q_{1} \\ \lambda_{q 1} & Q_{1} \leq Q_{g i} \leq 0 \\ \lambda_{q 0} & 0 \leq Q_{g i} \leq Q_{2} \\ \lambda_{q 0}+\left(a Q_{g i}+b\right) & Q_{2} \leq Q_{g i} \leq Q_{\max }\end{cases}
$$

The bidding model can encourage generator traders to join bidding of reactive power and make them reclaim their cost reasonably and take part in reactive power market positively, in order to make system get enough reactive power resources and satisfy the demand of reactive power load and provide voltage support. For a given unit, $\lambda_{q 0}$ should have a fixed value, because the investment cost of reactive power is lower oppositely, $\lambda_{q 0}$ can be prescribed maximal value to prevent gamble behaviors of generator 
traders and ensure the stability of reactive power market. Under the condition of invariable active power, reactive power's regulation of generator is an effective way to regulate voltage, which need not have extra expenditure, so describing $\lambda_{q 0}$ as a lesser value is not only accord with the characters of reactive power's generation but also can make system procure more reactive power from generator units; not only can make generator units reclaim their cost of reactive power but also decrease expenditure of reactive power ancillary services effectively, and accordingly decrease the reactive power expenditure that users bear, utilize resources adequately, to realize maximal social benefits [1].

\section{B. Cost of reactive compensators}

The charge for using reactive compensators is assumed proportional to the amount of the reactive power purchased and can be expressed as:

$$
C_{s h j}\left(Q_{s h j}\right)=r_{s h j i} Q_{s h j i}
$$

Where $r_{s h j i}$ and $Q_{s h j}$ are the reactive cost and the amount purchased, respectively, at location $\mathrm{j}$. The production cost of a compensator is assumed as its capital investment return, which can be expressed as its depreciation rate. For example, if the investment cost of a reactive compensator is $36000 \$ / M V A R$, and its average working rate and life span are $3 / 4$ and 35 years, respectively, the cost or depreciation rate of the compensator can be calculated as:

$$
r_{s h j}=\frac{36000}{35 \times 365 \times 24 \times \frac{3}{4}}=0.15655
$$

\subsection{Constraints}

In order to accommodate the market solution with actual power system condition, the operational constrains of the power system should be included into the model provided for reactive power procurement. The constraints of mentioned market are as follows:

\section{A. Constraint of Power Balance}

$$
\begin{aligned}
& \left(P_{g i}^{0}+\Delta P_{g i}\right)-P_{d i}^{0}=\sum_{j}^{N_{b u s}}\left|V_{i}\right|\left|V_{j}\right|\left|Y_{i, j}\right| \cdot \cos \left(\theta_{i, j}+\delta_{j}-\delta_{i}\right) \\
& Q_{g i}+Q_{s h i}-\tan \left(\varphi_{d i}^{0}\right) \cdot P_{d i}^{0}=-\sum_{j}^{N_{\text {bus }}}\left|V_{i}\right|\left|V_{j}\right|\left|Y_{i, j}\right| \cdot \sin \left(\theta_{i, j}+\delta_{j}-\delta_{i}\right)
\end{aligned}
$$

Where $\left|V_{i}\right|$ and $\delta_{i}$ represent the voltage magnitude and angle at node i. $\left|Y_{i, j}\right|$ and $\theta_{i, j}$ are the magnitude and angle of the admittance connecting bus $\mathrm{i}$ to bus $\mathrm{j} . \varphi_{d i}^{0}$ is the phase angle of the load at bus i.

\section{B. Voltage Stability Criteria}

Since, increase in power generation and consumption, conduct the system to instability margin so it is important for the isolated system operator to be aware of stability condition and distance to instability margin. In order to make decision the favorite criteria is the one which gives the exact distance to instability margin to the operator. Voltage Stability Criteria is able to estimate the exact distance to instability margin by the use of the direction of increase in power generation and consumption. It is supposed in this paper that the amount of power generation and consumption are increased proportional to the primary amount of them. In this approach, the voltage stability margin is incorporated in to the power flow equations to maintain the power system in a secure operating state where a sudden load variation or even a contingency occurs.

Various OPF based approaches to include voltage stability criteria as a security constraint are discussed in [20] in detail. Referring to [21], one way to ensure of voltage stability in power system operation is to dispatch the active and reactive power in such a way that sufficient safety margin exist to the collapse point. Beyond the collapse point, the power flow equations have no feasible solution. The voltage stability margin is usually measured in per unit MW and defined as the minimum distance from current operating point to the collapse point. This concept is graphically depicted in Fig. 3.

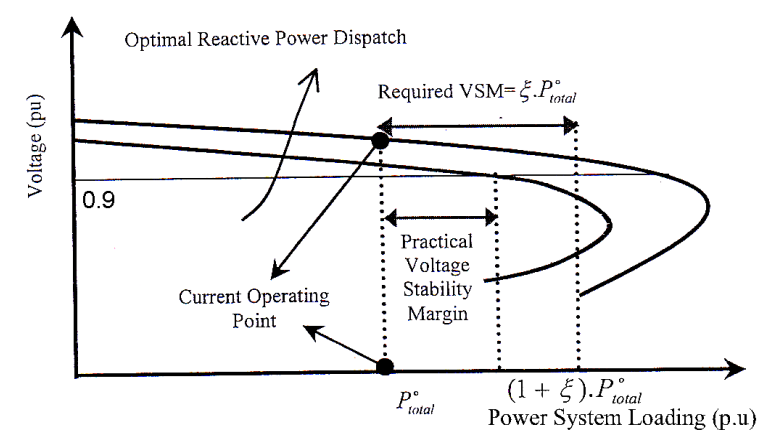

Fig. 3. Pictorial definition of voltage stability margins and loading factor

In this figure, $\xi$ is the loading factor of the system and $P_{\text {total }}^{0} \quad$ stands for the present operating point of the system and VSM measures the voltage stability margin. The VSM always refers to the theoretical stability margin because no capacity or voltage constraints of the network are involved in its calculation. Instead, practical voltage stability margin (PVSM) is widely used as practical implementation of the concept of voltage stability in power systems. PVSM is defined as the maximum load that can be added to the current operating point while no capacity or security limits are violated. In other words this figure shows that increase in total power by the amount of $\xi \cdot P_{\text {total }}^{0}$ does not make voltage collapse in the system. Consequently, it can be incorporate into the power flow equations and OPF formulation as following:

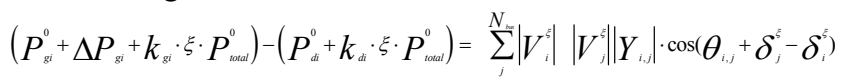




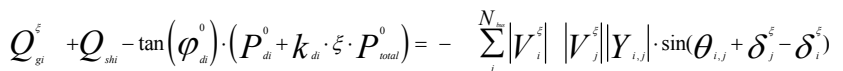

In Eqn. (6) and (7) parameters which are shown by superscript $(\xi)$ are the amount gained after increase in load. In order to simulate sudden load variation or increase in power generation and consumption, different directions can be selected to determine distribution coefficients. These coefficients are proposed by Eqn. (8) to (10).

$$
\begin{gathered}
k_{d i}=\frac{P_{d i}^{0}}{P_{\text {total }}^{0}} \\
k_{g i}=\frac{P_{g i}^{0}+\Delta P_{g i}}{P_{\text {total }}^{0}} \\
P_{\text {total }}^{0}=\sum_{i=1}^{N_{D}} P_{d i}^{0}
\end{gathered}
$$

\section{Active and Reactive Power generation/consumption capacity}

Generation and absorption capacity of reactive power and active power output of generators is limited as fallow:

$$
\begin{gathered}
P_{g i}^{\min } \leq P_{g i} \leq P_{g i}^{0}+\Delta P_{g i} \\
P_{d j}^{\min } \leq P_{d j} \leq P_{d j}^{0} \\
Q_{g i}^{\min } \leq Q_{g i} \leq Q_{g i}^{\max } ; Q_{g i}^{\min } \leq Q_{g i}^{\xi} \leq Q_{g i}^{\max } \\
Q_{s h i}^{\min } \leq Q_{s h i} \leq Q_{s h i}^{\max }
\end{gathered}
$$

Where $P_{g i}^{0}$ and $P_{d j}^{0}$ are the quantities of active power obtained through execution of the auction-based electricity market. The generators are allowed to offer some extra part of active power, namely $\Delta P_{g i}$ for compensation of the transmission losses. $Q_{g i}^{\max }$ and $Q_{g i}^{\min }$ are the maximum capacity of reactive power generation/absorption of generators at bus i respectively.

\section{Bus Voltage Limits}

$$
V_{i}^{\min } \leq V_{i} \leq V_{i}^{\max } ; V_{i}^{\min } \leq V_{i}^{\xi} \leq V_{i}^{\max }
$$

\section{E. Constraint of Transformer Ratio}

$$
T_{k}^{\min } \leq T_{k} \leq T_{k}^{\max }
$$

Where $T_{k}^{\min }$ and $T_{k}^{\max }$ are the minimal and maximal allowable range of ratio of Tap Changer respectively.

\section{Results and Discussion}

A genetic algorithm (GA) and particle swarm optimization (PSO) method are implemented in MATLAB programming language 7.6 to solve the problem of optimum allocation of reactive power in power systems under open market environment. For verifying the validity of the proposed model, it is tested on IEEE-30 bus system. The programs were run on a $3.2 \mathrm{GHz}$, Pentium-IV, with $512 \mathrm{MB}$ RAM PC.

- Parameters for the GA algorithm:

Population Size $=32$

Maximum Iterations $=200$

Crossover rate $=0.8$

Mutation rate $=0.01$

Crossover parameter $=0.5$

- Parameters for the PSO algorithm:

Population Size $=32$

Learning factors: $C_{1}=C_{2}=2.0$

Weighting factor: $\omega^{\max }=0.9, \omega^{\min }=0.4$

Maximum Iterations $=200$

The IEEE-30-bus power system consists of 41 branches, 6 generator-buses and 22 load-buses, of which four branches are under load-tap-setting transformer branches and four static VAR compensators [22]. It is shown in appendix. The categories, sites and limits of control variables are listed as follow:

1. Continuous AVR values of generator 1, 2, 5, 8, 11 and 13, the upper and lower limit is set to $0.9 \mathrm{pu}$ and $1.1 \mathrm{pu}$. Each generator unit proposes its bidding curve formulated with Eqn.(2). The reactive power bidding curve parameters of generator units are proposed in Table 1. Reactive power values are expressed in p.u. And the price of the losses is $10 \$ / P u(M W)$.

2. Discrete tap of transformers in lines 6-9, 6-10, 4-12 and $27-28$, the upper and lower limit is set to $0.9 \mathrm{pu}$ and $1.1 \mathrm{pu}$, which have 20 tap positions.

3. There are four static VAR compensators installed at bus $10,15,18$ and 24 with maximal values of output of $0.25 \mathrm{pu}$. It is assumed that the first two have presented the reactive power prices as $25 \$ / p . u(M V A R)$ and the last two $15 \$$ p.u (MVAR).

Table 1. Reactive power-bidding curve parameters of generators

\begin{tabular}{ccccccccc}
\hline $\begin{array}{c}\text { Gen. } \\
\text { Bus } \\
\text { No. }\end{array}$ & $\lambda_{q 1}$ & $\lambda_{q 0}$ & $a^{\prime}$ & $b^{\prime}$ & $a$ & $b$ & $Q_{1}$ & $Q_{2}$ \\
1 & 3.6 & 2.8 & -15 & -1.2 & 8 & -1.2 & -0.08 & 0.15 \\
2 & 3.4 & 2.6 & -15 & -1.8 & 9 & -0.9 & -0.12 & 0.1 \\
5 & 3.6 & 2.8 & -15 & -1.2 & 8 & -0.96 & -0.08 & 0.12 \\
8 & 3.4 & 2.6 & -14 & -1.68 & 9 & -0.9 & -0.12 & 0.1 \\
11 & 3.8 & 3 & -16 & -1.6 & 7 & -0.35 & -0.1 & 0.05 \\
13 & 3.8 & 3 & -16 & -1.6 & 7 & -0.35 & -0.1 & 0.05 \\
\hline
\end{tabular}




\subsection{Optimal allocation of reactive power:}

In this case each generator unit proposes its reactive power bidding curve and it is assumed that the voltage stability criteria has not considered. Therefore the loading factor $\xi$ and $\tau_{\text {gqi }}$ in Eqn.(1) is equal to zero. Table 2 shows final allocation of generator's active power and compares the results of both optimization methods. Final amount of reactive power of generators and static VAR compensators is showed in Table 3. Final tap position of transformers is obvious in Table 4. Consequently, Table V compares two methods and shows total active power of generators, active power losses and its cost, total reactive power obtained from reactive sources and their costs, there is also total costs obtained from optimization methods. As it is obvious from Table 5 the PSO algorithm has achieved better consequences in compare with GA, the former has smaller power loss and more important it gained better cost amount as total cost. Therefore, the PSO method is used in following section to achieve more accurate results.

Table 2. Final allocation of generator's active power (P.U.)

\begin{tabular}{|c|c|c|c|c|}
\hline variables & $\begin{array}{l}\text { Lower } \\
\text { limit }\end{array}$ & $\begin{array}{l}\text { Upper } \\
\text { limits }\end{array}$ & Final state (GA) & Final state (PSO) \\
\hline$P_{g 1}$ & 0.5 & 2 & 1.864 & 1.926 \\
\hline$P_{g 2}$ & 0.1 & 1 & 0.1 & 0.1 \\
\hline$P_{g 5}$ & 0.1 & 1 & 0.196 & 0.343 \\
\hline$P_{g 8}$ & 0.1 & 1 & 0.248 & 0.196 \\
\hline$P_{g 11}$ & 0.1 & 1 & 0.243 & 0.159 \\
\hline$P_{g 13}$ & 0.1 & 1 & 0.243 & 0.159 \\
\hline
\end{tabular}

Table 3. Final allocation of reactive power of generators and SVCs (P.U.)

\begin{tabular}{ccccc}
\hline variables & $\begin{array}{r}\text { Lower } \\
\text { limit }\end{array}$ & $\begin{array}{c}\text { Upper } \\
\text { limit }\end{array}$ & $\begin{array}{c}\text { Final state } \\
(\mathrm{GA})\end{array}$ & $\begin{array}{c}\text { Final state } \\
(\mathrm{PSO})\end{array}$ \\
$Q_{g 1}$ & -0.2 & 1 & 0.15 & 0.208 \\
$Q_{g 2}$ & -0.2 & 0.5 & 0.1 & 0.142 \\
$Q_{g 5}$ & -0.2 & 1 & 0.12 & 0.133 \\
$Q_{g 8}$ & -0.2 & 0.5 & 0.1 & 0.139 \\
$Q_{g 11}$ & -0.2 & 0.5 & 0.146 & 0.05 \\
$Q_{g 13}$ & -0.2 & 0.5 & 0.146 & 0.05 \\
$Q_{s h 10}$ & 0 & 0.25 & 0.032 & 0.039 \\
$Q_{s h 15}$ & 0 & 0.25 & 0.026 & 0.041 \\
$Q_{s h 18}$ & 0 & 0.25 & 0.121 & 0.094 \\
$Q_{s h 24}$ & 0 & 0.25 & 0.097 & 0.087 \\
\hline
\end{tabular}

Table 4. Final tap positions of transformers (P.U.)

\begin{tabular}{ccccc}
\hline variables & Lower limit & Upper limit & $\begin{array}{c}\text { Final state } \\
\text { (GA) }\end{array}$ & $\begin{array}{c}\text { Final state } \\
\text { (PSO) }\end{array}$ \\
$T_{6-9}$ & 0.9 & 1.1 & 1.1 & 1.04 \\
$T_{6-10}$ & 0.9 & 1.1 & 0.96 & 1.05 \\
$T_{4-12}$ & 0.9 & 1.1 & 1.05 & 0.9 \\
$T_{27-28}$ & 0.9 & 1.1 & 0.9 & 1.01 \\
\hline
\end{tabular}

\subsection{Reactive power market consequences with em- phasis on voltage stability criteria:}

In this case the optimal allocation of reactive power is investigated with emphasis on voltage stability criteria. As it was declared before, in this paper, it is supposed that the amount of power generation and consumption are varied proportional to the primary amount of them. Reactive power market consequences by the assumption of $\tau_{g q i}=0.5$ for the different amount of loading factor $\xi$ from 0.0 to 0.2 are proposed. Fig. 4 shows the cost of reactive, cost of loss and total cost which are obtained from PSO method. It is observed that by increase in voltage stability criteria the reactive power cost increases. The process of reactive power variation of generators and static VAR compensators are showed in Fig. 5 and Fig. 6 respectively. These figures confirm that the proposed model tries to prepare reactive power from the cheapest resources.

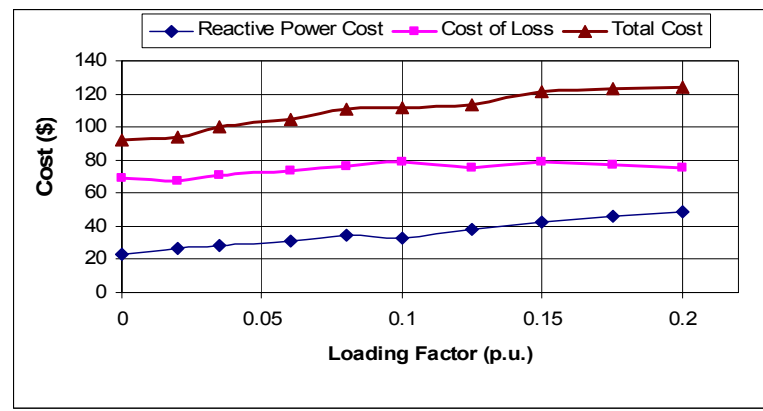

Fig. 4. Variation of cost function by increase in voltage stability criteria

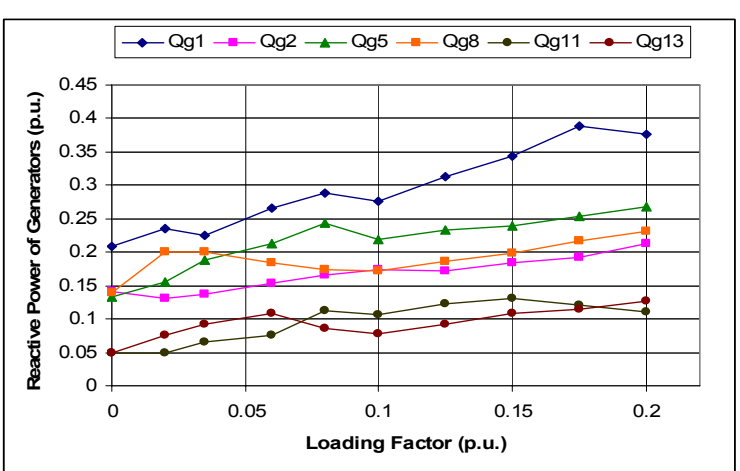

Fig. 5. Reactive power variation of generators by increase in voltage stability criteria 
Table 5. comparisons of optimal results for two methods

\begin{tabular}{|c|c|c|c|c|c|c|}
\hline Metho & $P_{g i}$ & $\sum Q_{g k s h}(p$ & Loss $\{p u$ & $\begin{array}{c}V_{G} \lambda_{g i}\left(Q_{g i}\right) \\
(\$)\end{array}$ & $\begin{array}{c}\sum_{j=1}^{N_{s h}} C_{s h j}\left(Q_{s h j}\right) \\
(\$)\end{array}$ & $\begin{array}{l}\text { Active } \\
\text { Power Total } \\
\text { Losses Cost } \\
\text { Cost }\end{array}$ \\
\hline GA & 2.894 & 1.038 & 0.0499 & 18.144 & 4.72 & 69.01892 .482 \\
\hline PSO & 2.883 & 0.983 & 0.0491 & 18.097 & 4.715 & 67.97491 .356 \\
\hline
\end{tabular}

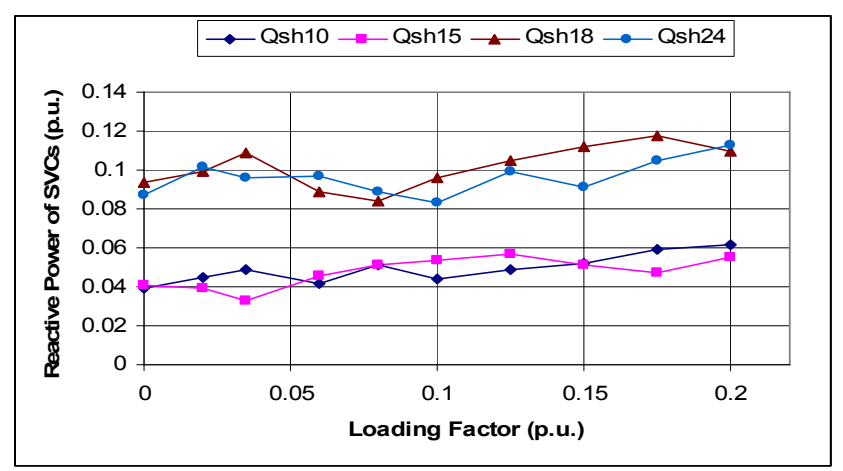

Fig. 6. Reactive power variation of SVCs by increase in voltage stability criteria

\section{Conclusion}

In this paper an approach was presented for optimal allocation of reactive power in deregulated electricity market. The proposed model minimizes reactive support cost as an economic aspect and insures the voltage security as a technical constraint. In this approach, the voltage stability margin is incorporated in to the power flow equations to maintain the power system in a secure operating state where a sudden load variation or even a contingency occurs. Based on analysis of generators' production cost of reactive power, a segmented bidding model of generators' reactive power generation is exerted. The proposed method specifies the active and reactive power of generators and the reactive power of static VAR compensators and the variation of transformers tap changer as well as their cost. In this paper both active and reactive power flow is accomplished while optimization. Each reactive power supports proposes its reactive power capacity and its cost by a clear function so there are a transparent competition between reactive generators. Finally, a genetic algorithm (GA) and particle swarm optimization (PSO) method are compared to solve the problem. The performance of the proposed model is validated on IEEE-30 bus system. Results demonstrate that the algorithm is well competent in achieving the near optimal allocation of reactive power under practical constraints and price based conditions.

\section{Acknowledgements}

This work was supported by the K. N. Toosi University of technology, Tehran, Iran.

\section{Appendix}

Table A.1. Bus data of IEEE-30 bus power system

\begin{tabular}{|c|c|c|c|c|c|}
\hline Bus No & $\mathrm{Pd}$ & Qd & Bs & Gs & BaseKV \\
\hline 1 & 0 & 0 & 0 & 0 & 132 \\
\hline 2 & 21.7 & 12.7 & 0 & 0 & 132 \\
\hline 3 & 2.4 & 1.2 & 0 & 0 & 132 \\
\hline 4 & 7.6 & 1.6 & 0 & 0 & 132 \\
\hline 5 & 94.2 & 19 & 0 & 0 & 132 \\
\hline 6 & 0 & 0 & 0 & 0 & 132 \\
\hline 7 & 22.8 & 10.9 & 0 & 0 & 132 \\
\hline 8 & 30 & 30 & 0 & 0 & 132 \\
\hline 9 & 0 & 0 & 0 & 0 & 1 \\
\hline 10 & 5.8 & 2 & 19 & 0 & 33 \\
\hline 11 & 0 & 0 & 0 & 0 & 11 \\
\hline 12 & 11.2 & 7.5 & 0 & 0 & 33 \\
\hline 13 & 0 & 0 & 0 & 0 & 11 \\
\hline 14 & 6.2 & 1.6 & 0 & 0 & 33 \\
\hline 15 & 8.2 & 2.5 & 0 & 0 & 33 \\
\hline 16 & 3.5 & 1.8 & 0 & 0 & 33 \\
\hline 17 & 9 & 5.8 & 0 & 0 & 33 \\
\hline 18 & 3.2 & 0.9 & 0 & 0 & 33 \\
\hline 19 & 9.5 & 3.4 & 0 & 0 & 33 \\
\hline 20 & 2.2 & 0.7 & 0 & 0 & 33 \\
\hline 21 & 17.5 & 11.2 & 0 & 0 & 33 \\
\hline 22 & 0 & 0 & 0 & 0 & 33 \\
\hline 23 & 3.2 & 1.6 & 0 & 0 & 33 \\
\hline 24 & 8.7 & 6.7 & 4.3 & 0 & 33 \\
\hline 25 & 0 & 0 & 0 & 0 & 33 \\
\hline 26 & 3.5 & 2.3 & 0 & 0 & 33 \\
\hline 27 & 0 & 0 & 0 & 0 & 33 \\
\hline 28 & 0 & 0 & 0 & 0 & 132 \\
\hline 29 & 2.4 & 0.9 & 0 & 0 & 33 \\
\hline 30 & 10.6 & 1.9 & 0 & 0 & 33 \\
\hline
\end{tabular}


Table A.2. Branch data of IEEE-30 bus power system

\begin{tabular}{|c|c|c|c|c|}
\hline From Bus & To Bus & b (p.u.) & x (p.u.) & r (p.u.) \\
\hline 1 & 2 & 0.0528 & 0.0575 & 0.0192 \\
\hline 1 & 3 & 0.0408 & 0.1652 & 0.0452 \\
\hline 2 & 4 & 0.0368 & 0.1737 & 0.057 \\
\hline 3 & 4 & 0.0084 & 0.0379 & 0.0132 \\
\hline 2 & 5 & 0.0418 & 0.1983 & 0.0472 \\
\hline 2 & 6 & 0.0374 & 0.1763 & 0.0581 \\
\hline 4 & 6 & 0.009 & 0.0414 & 0.0119 \\
\hline 5 & 7 & 0.0204 & 0.116 & 0.046 \\
\hline 6 & 7 & 0.017 & 0.082 & 0.0267 \\
\hline 6 & 8 & 0.009 & 0.042 & 0.012 \\
\hline 6 & 9 & 0 & 0.208 & 0 \\
\hline 6 & 10 & 0 & 0.556 & 0 \\
\hline 9 & 11 & 0 & 0.208 & 0 \\
\hline 9 & 10 & 0 & 0.11 & 0 \\
\hline 4 & 12 & 0 & 0.256 & 0 \\
\hline 12 & 13 & 0 & 0.14 & 0 \\
\hline 12 & 14 & 0 & 0.2559 & 0.1231 \\
\hline 12 & 15 & 0 & 0.1304 & 0.0662 \\
\hline 12 & 16 & 0 & 0.1987 & 0.0945 \\
\hline 14 & 15 & 0 & 0.1997 & 0.221 \\
\hline 16 & 17 & 0 & 0.1923 & 0.0524 \\
\hline 15 & 18 & 0 & 0.2185 & 0.1073 \\
\hline 18 & 19 & 0 & 0.1292 & 0.0639 \\
\hline 19 & 20 & 0 & 0.068 & 0.034 \\
\hline 10 & 20 & 0 & 0.209 & 0.0936 \\
\hline 10 & 17 & 0 & 0.0845 & 0.0324 \\
\hline 10 & 21 & 0 & 0.0749 & 0.0348 \\
\hline 10 & 22 & 0 & 0.1499 & 0.0727 \\
\hline 21 & 22 & 0 & 0.0236 & 0.0116 \\
\hline 15 & 23 & 0 & 0.202 & 0.1 \\
\hline 22 & 24 & 0 & 0.179 & 0.115 \\
\hline 23 & 24 & 0 & 0.27 & 0.132 \\
\hline 24 & 25 & 0 & 0.3292 & 0.1885 \\
\hline 25 & 26 & 0 & 0.38 & 0.2544 \\
\hline 25 & 27 & 0 & 0.2087 & 0.1093 \\
\hline 28 & 27 & 0 & 0.396 & 0 \\
\hline 27 & 29 & 0 & 0.4153 & 0.2198 \\
\hline 27 & 30 & 0 & 0.6027 & 0.3202 \\
\hline 29 & 30 & 0 & 0.4533 & 0.2399 \\
\hline 8 & 28 & 0.0428 & 0.2 & 0.0636 \\
\hline
\end{tabular}

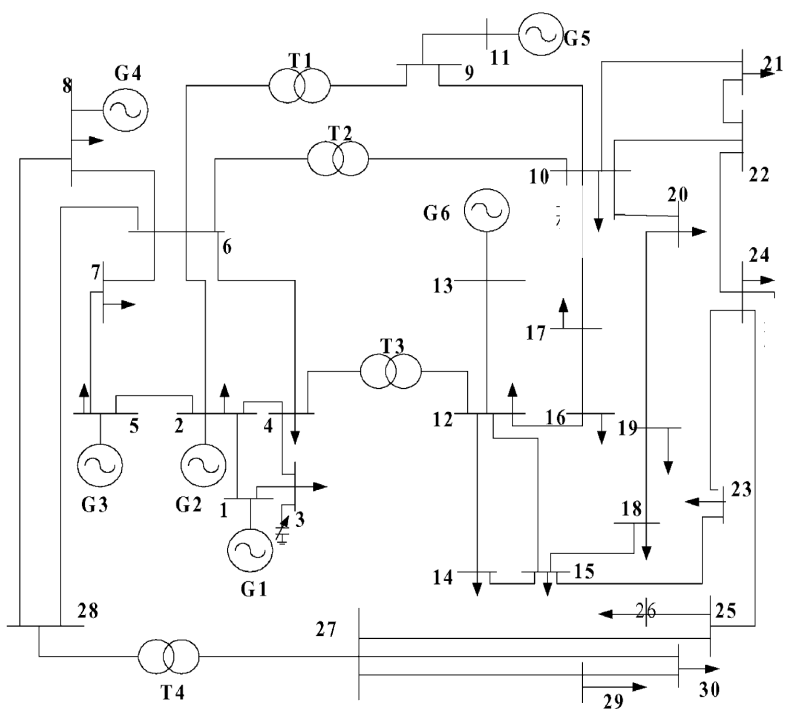

Fig. A.1. IEEE 30-bus power system.

\section{References}

[1] Nidul Sinha, Loi Lei Lai and Palash Kumar Ghosh, "GA Based Algorithm for Optimum Allocation of Reactive Power under Deregulated Environment," IEEE DRPT2008 Conference, April 2008 Nanjing China.

[2] S.K. Parida, S.N. Singh and S.C. Srivastava, "Voltage Security Constrained Localized Reactive Power Market," 0-7803-9525-5/06/\$20.00 (C2006 IEEE.

[3] El-Samahy, K. Bhattacharya, and C. A. Cañizares, "A Unified Framework for Reactive Power Management in Deregulated Electricity Markets," IEEE, PSCE 2006, pp. 901-907.

[4] Alex D. Papalexopoulos, George A. Angelidis, "Reactive Power Management and Pricing in the California Market," IEEE MELECON, Benalmádena (Malaga), Spain, May 2006, pp. 902-905.

[5] Lamont JW, Fu J., "Cost analysis of reactive power support,” IEEE Trans Power Syst. 1999;14(3):890 896.

[6] X.J. Lin, C.W. Yu and C.Y. Chung, "Pricing of reactive Power Support Ancillary Services," IEE proc.Gener. Transm. Distrib., vol. 152, no. 5, September2005, pp. 616-622.

[7] Baughman ML, Siddiqi SN., "Real time pricing of reactive power: theory and case study results," IEEE Trans Power Syst 1991; 6(1):23-9.

[8] Li YZ, David AK., "Wheeling rates of reactive power flow under marginal cost pricing," IEEE Trans. Power Syst. 1994; 9(3):1263-9

[9] Bhattacharya K, Zhong J., "Reactive power as an ancillary service," IEEE Trans Power Syst 2001; 16(2):294-300.

[10] Zhong J, Bhattacharya K., "Toward a competitive market for reactive power," IEEE Trans Power Syst 2002;17(4):1206-15. 
[11] Hao S., "A reactive power management proposal for transmission operators," IEEE Trans Power Syst 2003; 18(4):1374-80.

[12] Silva EL, Hedgecock JJ, Mello JCO, Luz JCF., "Practical cost-based approach for the voltage ancillary service," IEEE Trans Power Syst 2001; 16(4): 806-12.

[13] X.J. Lin, C.W. Yu, A.K. David, C.Y. Chung, H. Wu, $\mathrm{N} . \mathrm{Xu}$, "A novel market-based reactive power management scheme," ELSEVIER Electrical Power and Energy Systems 28 (2006) 127-132.

[14] R.S. Salgado, and M.R. Irving, "Framework for Analysis of reactive Power Dispatch in Energy Pools," IEE Proc.-Gener. Transm. Distrib., vol. 151, no. 2, March 2004, pp. 1047-1052.

[15] Wen-Chen Chu, Bin-Kwie Chen and chung Liao, "Allocation the Cost of Reactive Power purchased in an Ancillary Service Markets by Modified Y-Bus Matrix Method," IEEE Transaction on Power Systems, vol. 19, no. 1, February 2004, pp. 174-179.

[16] Julian Barquin Gil, and et al, "Reactive Power Pricing: a Conceptual Framework Remuneration and charging Procedure," IEEE Transaction on Power Systems, vol. 15, no. 2, May 2000, pp. 483-489.

[17] X.J. Lin and A.K, David and C.W. Yu, "Reactive Power optimization with Voltage stability consideration in Power Market Systems," IEE proc.-Gener. Transm. Distrib., vol. 150, no. 3, July 2003, pp. 305310.

[18] M.J. Rider and V.L. Paucar, "Application of Nonlinear Reactive Power Pricing Model for Competitive Electric Markets," IEE proc.-Gener. Transm. Distrib. vol. 151, no. 3, May 2004, pp. 407-414.

[19] Li Gengyin, Zhou Ming, Yang Juan, and Sun Liying, "Research on Procurement Strategy of Reactive Power as Auxiliary Service in Power Markets," IEEE International Conference on Electric Utility Deregulation, Restructuring and Power Technologies (DRPT2004), pp. 349-354, April 2004.

[20] W. Rosehart, C. Cafiizares, and V. H. Quintana, “Optimal Power Flow Incorporating Voltage Collapse Constraints," Proc. of the 1999 IEEE/PES Summer Meeting, Edmonton, Alberta, July 1999, pp. 820-825.

[21] B. Mozafari, T.amraee, A. M. Ranjbar, M. Mirjafari, and A. R. Shirani, "Optimal Reactive Power Dispatch in a Joint Active / Reactive Market Model," IEEE, International Conference on Power System Technology, 2006.

[22] Q. H. Wu, Y. J. Cao, and J. Y. Wen, "Optimal reactive power dispatch using an adaptive genetic algorithm," Int. J. Elect. Power Energy Syst., vol. 20, pp. 563-569, Aug. 1998.

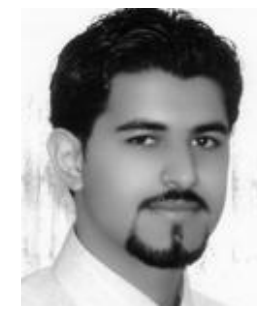

Mahdi Rajabzadeh $\mathrm{He}$ was born in Masal in Iran, in 1983. He received his BSc degree from Guilan University, Iran in 2005 and is a MSc student in K. N. Toosi University of Technology, Tehran, Iran. He has worked on power system restoration and system security as his important researches. And now he is studying on reactive power pricing and management in deregulated environment. mahdi_rajabzadeh@ee. kntu.ac.ir

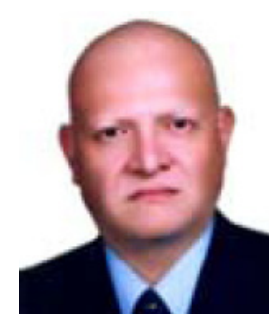

Masoud AliAkbar Golkar He was born in Tehran Iran in 1954. He received his BSc degree from Sharif University of Technology Tehran-Iran in 1977, MSc from The Oklahoma State University USA in 1979 and his PhD degree from the Imperial College of Science, Technology, and Medicine (The University of London, UK) in 1986, all in Electrical Engineering (Power Systems). His employment experience included working as an Associate Professor at The K N Toosi University, Tehran, Advisor to Tehran Electricity Board, Head of research group at Electric Power Research Center in Tehran .Now he is an Associate Professor at K N Toosi University of Technology in Tehran, Iran. Golkar@eetd. kntu.ac.ir 